\title{
Bag-of-Features Image Indexing and Classification in Microsoft SQL Server Relational Database
}

\author{
Marcin Korytkowski, Rafał Scherer, Paweł Staszewski, Piotr Woldan \\ Institute of Computational Intelligence \\ Częstochowa University of Technology \\ al. Armii Krajowej 36, 42-200 Czȩstochowa, Poland \\ Email: marcin.korytkowski@ iisi.pcz.pl, rafal.scherer@iisi.pcz.pl
}

\begin{abstract}
This paper presents a novel relational database architecture aimed to visual objects classification and retrieval. The framework is based on the bag-of-features image representation model combined with the Support Vector Machine classification and is integrated in a Microsoft SQL Server database.
\end{abstract}

Keywords-content-based image processing, relational databases, image classification

\section{INTRODUCTION}

Thanks to content-based image retrieval (CBIR) [1][2][3][4][5][6][7][8] we are able to search for similar images and classify them [9][10][11][12][13]. Images can be analyzed based on color representation [14][15][16], textures [17][18][19][20], shape [21][22][23] or edge detectors [24]. Recently, local invariant features have gained a wide popularity [25][26]|27][28][29]. The most popular local keypoint detectors and descriptors are SURF [30], SIFT [25] or ORB [31]. To find similar images to a query image, we need to compare all feature descriptors of all images usually by some distance measures. Such comparison is enormously time consuming and there is ongoing worldwide research to speed up the process. Yet, the current state of the art in the case of high-dimensional computer vision applications is not fully satisfactory. The literature presents countless methods and variants utilizing e.g. a voting scheme or histograms of clustered keypoints. They are mostly based on some form of approximate search. Recently, the bag-of-features (BoF) approach [32]|33||29]|34|[35] has gained in popularity. In the $\mathrm{BoF}$ method, clustered vectors of image features are collected and sorted by the count of occurrence (histograms). All individual descriptors or approximations of sets of descriptors presented in the histogram form must be compared. Such calculations are computationally expensive. Moreover, the $\mathrm{BoF}$ approach requires to redesign the classifiers when new visual classes are added to the system.

The paper deals with a visual query-by-example problem in relational databases. Namely, we developed a system based on Microsoft SQL Server which is able to classify a sample image or to return similar images to this image. Storing huge amount of undefined and unstructured binary data and its fast and efficient searching and retrieval is the main challenge for database designers. Examples of such data are images, video files etc. Users of world most popular relational database management systems (RDBMS) such as Oracle, MS SQL Server and IBM DB2 Server are not encouraged to store such

978-1-4799-8322-3/15/\$31.00 (c) 2015 IEEE data directly in the database files. The example of such an approach can be Microsoft SQL Server where binary data is stored outside the RDBMS and only the information about the data location is stored in the database tables. MS SQL Server utilizes a special field type called FileStream which integrates SQL Server database engine with NTFS file system by storing binary large object (BLOB) data as files in the file system. Microsoft SQL dialect (Transact-SQL) statements can insert, update, query, search, and back up FileStream data. Application Programming Interface provides streaming access to the data. FileStream uses operating system cache for caching file data. This helps to reduce any negative effects that FileStream data might have on the RDBMS performance. Filestream data type is stored as a varbinary (max) column with pointer to actual data which are stored as BLOBs in the NTFS file system. By setting the FileStream attribute on a column and consequently storing BLOB data in the file system, we achieve the following advantages:

- performance is the same as the NTFS file system and SQL Server cache is not burden with the Filestream data,

- Standard SQL statements such as SELECT, INSERT, UPDATE, and DELETE work with FileStream data; however, associated files can be treated as standard NTFS files.

In the proposed system, large image files are stored in a FileStream field. Unfortunately, despite using this technique, there does not exist a technology for fast and efficient retrieval of images based on their content in existing relational database management systems. Standard SQL language does not contain commands for handling multimedia, large text objects, and spatial data.

We designed a special type of field, in which a set of keypoints can be stored in an optimal way, as so-called UserDefined Type (UDT). Along with defining the new type of field, it is necessary to implement methods to compare its content. When designing UDT, various features must be also implemented, depending on implementing the UDT as a class or a structure, as well as on the format and serialization options. This could be done using one of the supported .NET Framework programming languages and the UDT can be implemented as a dynamic-link library (DLL), loaded in MS SQL Server. Another major challenge is to create a special database indexing algorithm, which would significantly speed up answering to SQL queries for data based on the newly de- 
fined field. As aforementioned, standard SQL does not contain commands for handling multimedia, large text objects and spatial data. Thus, communities that create software for processing such specific data types, began to draw up SQL extensions, but they transpired to be incompatible with each other. That problem caused abandoning new task-specific extensions of SQL and a new concept won, based on libraries of object types SQL99 intended for processing specific data applications. The new standard, known as SQL/MM (full name: SQL Multimedia and Application Packages), was based on objects, thus programming library functionality is naturally available in SQL queries by calling library methods. SQL/MM consists of several parts: framework - library for general purposes, full text - defines data types for storing and searching large amount of text, spatial - for processing geospatial data, still image - defines types for processing images and data mining data exploration. There are also attempts to create some SQL extensions using fuzzy logic for building flexible queries. In [36] possibilities of creating flexible queries and queries based on users examples are presented. It should be emphasized that the literature shows little efforts of creating a general way of querying multimedia data.

The main contribution and novelty of the paper is as follows:

- We present a novel system for content-based image classification built in a Microsoft SQL Server database,

- We created a special database indexing algorithm, which will significantly speed up answering to visual query-by-example SQL queries in relational databases.

The paper is organized as follows. Section II describes the proposed database system. Section III provides simulation results on the the PASCAL Visual Object Classes (VOC) 2012 dataset [37].

\section{SySTEM ARCHITECTURE AND RELATIONAL DATABASE STRUCTURE}

Our system and generally BoF can work with various image features. In the paper we use SIFT features as an example. To calculate SIFT keypoints we used the OpenCV library. We did not use functions from this library as a user defined functions (UDF) directly in the database environment because:

1) User Defined Functions can be written only in the same .NET framework version as the MS SQL Server (e.g. MS SQL Server was created based on .NET 4.0)

2) Calculations used to find image keypoints are very complex, thus running such computations directly on the database server causes the database engine to become unresponsive.

From the above-mentioned reasons, similarly as in the case of the Full Text Search technology, the most time-consuming computations are moved to the operating system as background system services of WCF (Windows Communication Foundation). WCF Data Service works as the REST architecture (Representational State Transfer) which was introduced by Roy T. Fielding in his PhD thesis [38]. Thanks to WCF technology, it

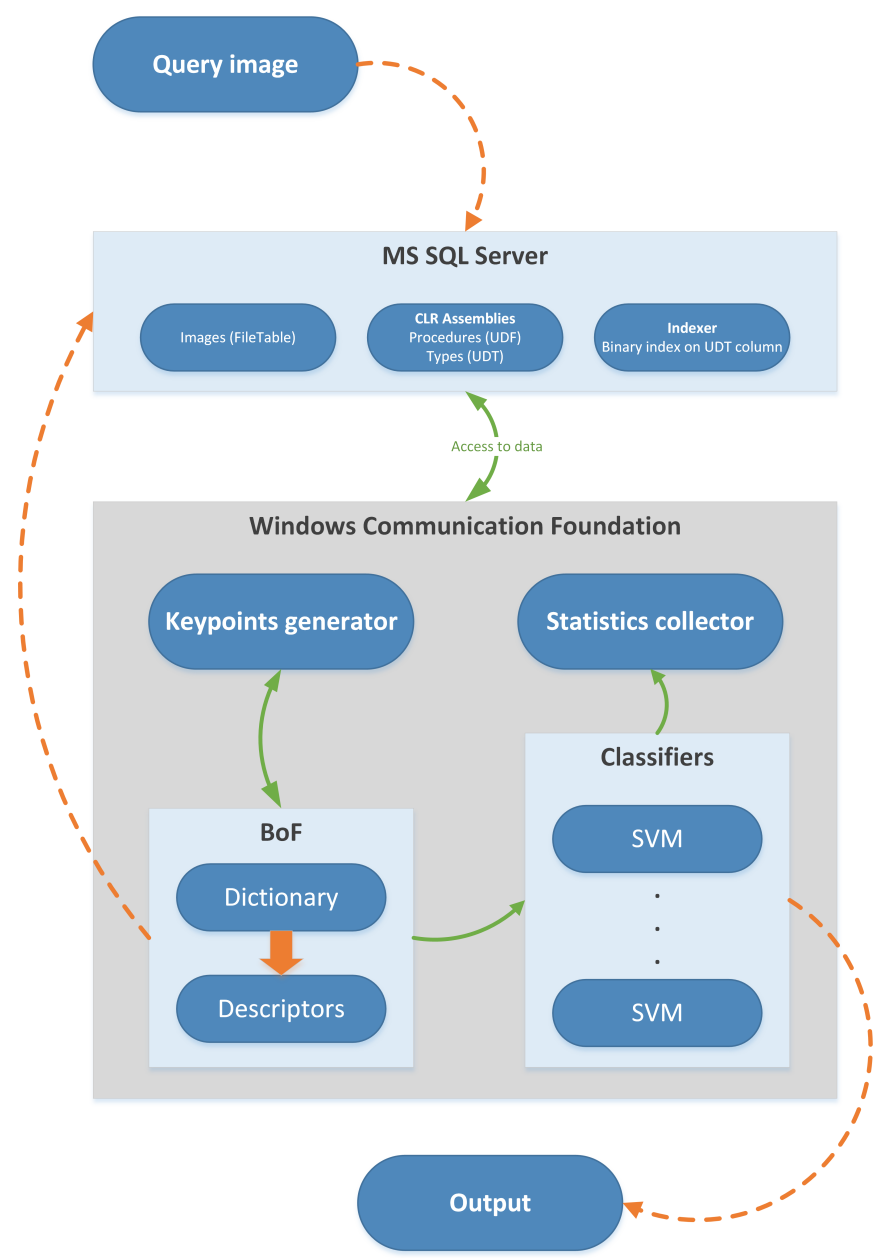

Fig. 1. System architecture

is relatively easy to set the proposed solution in the Internet. To store image local keypoints in the database, we created a User Defined Type (column sift_keypoints in SIFTS table). These values are not used in the classification of new query images. They are stored in case we need to identify a new class of objects in the existing images as having keypoint values, we would not have to generate keypoint descriptors again. Newly created type was created in C\# language as a CLR class and only its serialized form is stored in the database. The database stores also Support Vector Machine classifiers parameters in the SVMConfigs table. Such an approach allows running any time the service with learned parameters. Running the service in the operating system will cause reading SVM classifiers from the database. The Stats table is for collecting algorithm statistics, where the most important numbers are execution times for consecutive stages of the algorithm. The Images table is for storing membership of images for visual classes. Dictionaries table is responsible for storing keypoint clusters data, and these cluster parameters are stored in the DictionaryData field of UDT type:

public struct DictionaryData:

INullable, IB inarySerialize

\{

private bool null; 

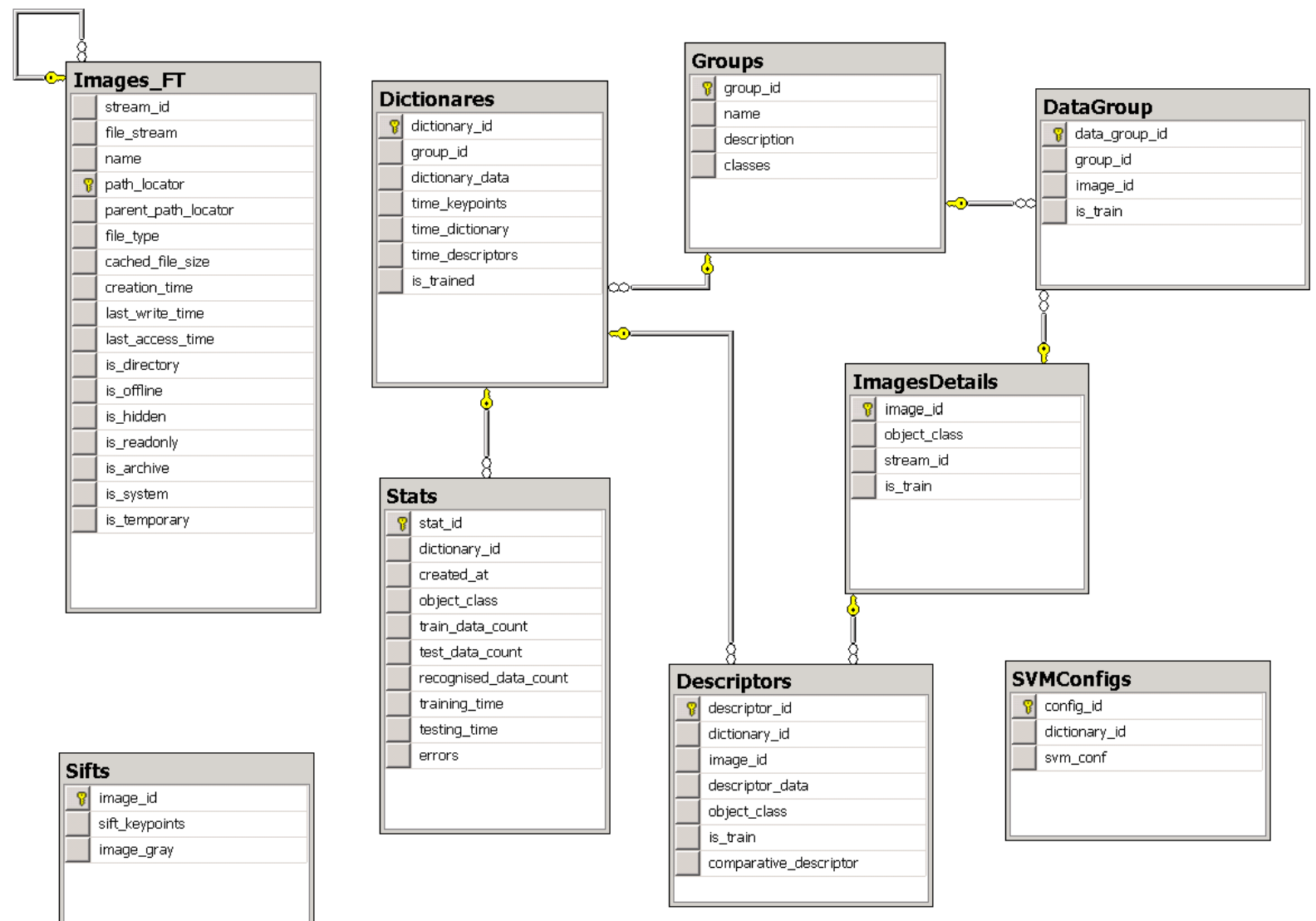

Fig. 2. Proposed database structure

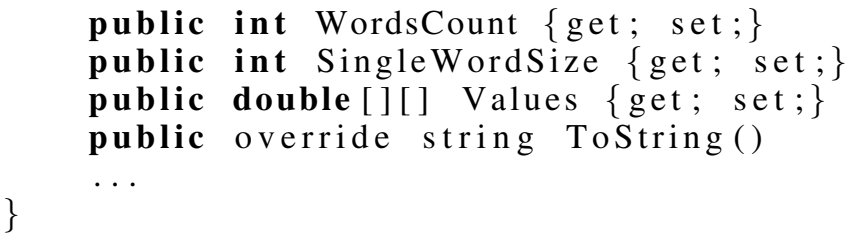

The WordsCount variable stores information about the number of words in the BoF dictionary, SingleWordSize variable value depends on the algorithm used to generate image keypoint descriptors, and in case of the SIFT algorithm, it equals 128. Two-dimensional matrix Values stores information regarding cluster centers. The system operates in two modes:

1) learning mode: Image keypoint descriptors are clustered to build a bag-of-features dictionary by the $k$-means algorithm. Cluster parameters are stored in DictionaryData variables. Next, image descriptors are created for subsequent images. They can be regarded as histograms of membership of image local keypoints to words from dictionaries. We use SIFTDetector method from the Emgu CV (http://www.emgu.com) library with the following signature: ComputeDescriptorsRaw (Image<Gray, byte>grayScaleImage, Image<Gray, byte> mask, Vectorofkeypoint keypoints). Obtained descriptors are then stored in the Descriptors table of
UDT type:

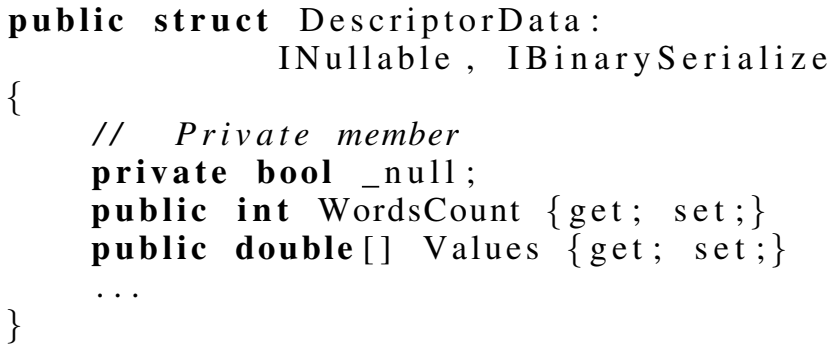

Using records from this table, learning datasets are generated for SVM classifiers to recognize various visual classes. These classifiers parameters are stored after the training phase in the SVMConfigs table.

2) Classification Mode: In the classification phase, the proposed system works fully automatically. After sending an image file to the Images_FT table, a service generating local interest points is launched. In the proposed approach, we use SIFT descriptors. Next, the visual descriptors are checked against membership to clusters stored in the database in the Dictionaries table and on this base, the histogram descriptor is created. To determine membership to a visual class we have to use this vector as the input for all SVM classifiers obtained in the learning phase. For the classification purposes, we extended SQL language and defined 


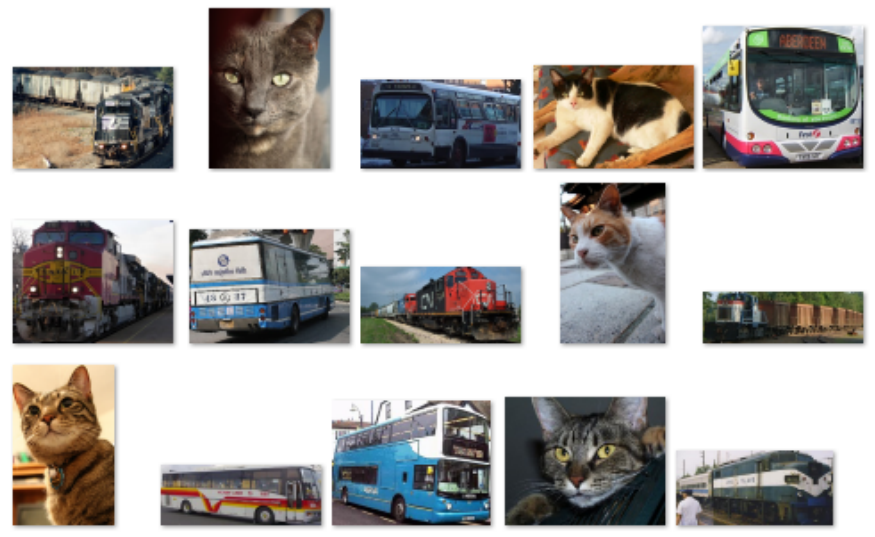

Fig. 3. Exemplary images from testing dataset

TABLE I. NUMERICAL SIMULATION RESULTS FOR VARIOUS BOF DICTIONARY SIZE.

\begin{tabular}{|l|l|l|l|l|l|l|}
\hline Words: & $\mathbf{4 0}$ & $\mathbf{5 0}$ & $\mathbf{8 0}$ & $\mathbf{1 0 0}$ & $\mathbf{1 3 0}$ & $\mathbf{1 5 0}$ \\
\hline Bus & $40 \%$ & $50 \%$ & $60 \%$ & $60 \%$ & $70 \%$ & $50 \%$ \\
\hline Cat & $90 \%$ & $80 \%$ & $50 \%$ & $80 \%$ & $80 \%$ & $80 \%$ \\
\hline Train & $0 \%$ & $0 \%$ & $10 \%$ & $20 \%$ & $10 \%$ & $10 \%$ \\
\hline Result: & $\mathbf{4 3 \%}$ & $\mathbf{4 3 \%}$ & $\mathbf{4 0 \%}$ & $\mathbf{5 3 \%}$ & $\mathbf{5 3 \%}$ & $\mathbf{4 7 \%}$ \\
\hline
\end{tabular}

GetClassofImage() method in C\# language and added it to the set of User Defined Functions. The argument of this method is the file identifier from the FileTable table.

Microsoft SQL Server constraints the sum of indexed columns to 900 bytes. Therefore, it was not possible to create an index on the columns constituting visual descriptors. To allow fast image searching of the Descriptors table, we created a field comparative_descriptor that stores descriptor value hashed by the MD5 algorithm. It allowed creating index on this new column, thus the time to find an image corresponding with the query image was reduced substantially.

\section{NUMERICAL SimUlations}

We tested the proposed method on three classes of visual objects taken from the PASCAL Visual Object Classes (VOC) dataset [37], namely: Bus, Cat and Train. We divided these three classes of objects into learning and testing examples. The testing set consists of $15 \%$ images from the whole dataset. Before the learning procedure we generated local keypoint vectors for all images from the Pascal VOC dataset using the SIFT algorithm. All simulations were performed on a Hyper$\mathrm{V}$ virtual machine with MS Windows Operating System (8 GB RAM, Intel Xeon X5650, $2.67 \mathrm{GHz}$ ). The testing set only contained images that had never been presented to the system during learning process.

The bag-of-features image representation model combined with the Support Vector Machine (SVM) classification was run five times for various dictionary sizes: $40,50,80,100$, 130 and 150 words. Dictionaries for the BoF were created using C++ language, based on the OpenCV Library [39]. The results of the $\mathrm{BoF}$ and SVM classification on the testing data are presented in Table I. The SQL queries responses are nearly real-time for even relatively large image datasets.

\section{CONCLUSION}

We presented a method that allows integrating relatively fast content-based image classification algorithm with relational database management system. Namely, we used bag of features, Support Vector Machine classifiers and special Microsoft SQL Server features, such as User Defined Types and CLR methods, to classify and retrieve visual data. Moreover, we created indexes to search for the same query image in large sets of visual records. Described framework allows automatic searching and retrieving images on the base of their content using the SQL language. The SQL responses are nearly realtime on even relatively large image datasets. The system can be extended to use different visual features or to have a more flexible SQL querying command set.

\section{ACKNOWLEDGMENT}

This work was supported by the Polish National Science Centre (NCN) under project number DEC2011/01/D/ST6/06957.

\section{REFERENCES}

[1] J. A. Daniel Carlos, Guimaraes Pedronette and R. da S. Torres, "A scalable re-ranking method for content-based image retrieval," Information Sciences, vol. 265, no. 0, pp. 91 - 104, 2014.

[2] P. Drozda, K. Sopyla, and P. Górecki, "Online crowdsource system supporting ground truth datasets creation," in Artificial Intelligence and Soft Computing - 12th International Conference, ICAISC 2013, Zakopane, Poland, June 9-13, 2013, Proceedings, Part I, 2013, pp. 532539.

[3] T. Kanimozhi and K. Latha, "An integrated approach to region based image retrieval using firefly algorithm and support vector machine," Neurocomputing, vol. 151, Part 3, no. 0, pp. 1099 - 1111, 2015.

[4] E. Karakasis, A. Amanatiadis, A. Gasteratos, and S. Chatzichristofis, "Image moment invariants as local features for content based image retrieval using the bag-of-visual-words model," Pattern Recognition Letters, vol. 55, no. 0, pp. $22-27,2015$.

[5] C.-H. Lin, H.-Y. Chen, and Y.-S. Wu, "Study of image retrieval and classification based on adaptive features using genetic algorithm feature selection," Expert Systems with Applications, vol. 41, no. 15, pp. 6611 - 6621, 2014.

[6] G.-H. Liu and J.-Y. Yang, "Content-based image retrieval using color difference histogram," Pattern Recognition, vol. 46, no. 1, pp. 188 198, 2013.

[7] S. Liu and X. Bai, "Discriminative features for image classification and retrieval," Pattern Recognition Letters, vol. 33, no. 6, pp. 744 - 751, 2012.

[8] E. Rashedi, H. Nezamabadi-pour, and S. Saryazdi, "A simultaneous feature adaptation and feature selection method for content-based image retrieval systems," Knowledge-Based Systems, vol. 39, no. 0, pp. $85-$ 94, 2013.

[9] M. Bazarganigilani, "Optimized image feature selection using pairwise classifiers," Journal of Artificial Intelligence and Soft Computing Research, vol. 1, no. 2, pp. 147-153, 2011.

[10] Y. Chang, Y. Wang, C. Chen, and K. Ricanek, "Improved imagebased automatic gender classification by feature selection," Journal of Artificial Intelligence and Soft Computing Research, vol. 1, no. 3, pp. 241-253, 2011.

[11] B. Karimi and A. Krzyzak, "A novel approach for automatic detection and classification of suspicious lesions in breast ultrasound images," Journal of Artificial Intelligence and Soft Computing Research, vol. 3, no. 4, pp. 265-276, 2013.

[12] N. Shrivastava and V. Tyagi, "Content based image retrieval based on relative locations of multiple regions of interest using selective regions matching," Information Sciences, vol. 259, no. 0, pp. 212 - 224, 2014. 
[13] J. Yang, K. Yu, Y. Gong, and T. Huang, "Linear spatial pyramid matching using sparse coding for image classification," in Computer Vision and Pattern Recognition, 2009. CVPR 2009. IEEE Conference on, June 2009, pp. 1794-1801.

[14] J. Huang, S. Kumar, M. Mitra, W.-J. Zhu, and R. Zabih, "Image indexing using color correlograms," in Computer Vision and Pattern Recognition, 1997. Proceedings., 1997 IEEE Computer Society Conference on, Jun 1997, pp. 762-768.

[15] S. Kiranyaz, M. Birinci, and M. Gabbouj, "Perceptual color descriptor based on spatial distribution: A top-down approach," Image Vision Comput., vol. 28, no. 8, pp. 1309-1326, Aug. 2010.

[16] G. Pass and R. Zabih, "Histogram refinement for content-based image retrieval," in Applications of Computer Vision, 1996. WACV '96., Proceedings 3rd IEEE Workshop on, Dec 1996, pp. 96-102.

[17] T. Chang and C.-C. Kuo, "Texture analysis and classification with treestructured wavelet transform," Image Processing, IEEE Transactions on, vol. 2, no. 4, pp. 429-441, Oct 1993.

[18] J. Francos, A. Meiri, and B. Porat, "A unified texture model based on a 2-d wold-like decomposition," Signal Processing, IEEE Transactions on, vol. 41, no. 8, pp. 2665-2678, Aug 1993.

[19] A. K. Jain and F. Farrokhnia, "Unsupervised texture segmentation using gabor filters," Pattern Recognition, vol. 24, no. 12, pp. 1167 - 1186, 1991.

[20] J. Śmietański, R. Tadeusiewicz, and E. Łuczyńska, "Texture analysis in perfusion images of prostate cancera case study," International Journal of Applied Mathematics and Computer Science, vol. 20, no. 1, pp. 149156, 2010.

[21] H. V. Jagadish, "A retrieval technique for similar shapes," SIGMOD Rec., vol. 20, no. 2, pp. 208-217, Apr. 1991.

[22] H. Kauppinen, T. Seppanen, and M. Pietikainen, "An experimental comparison of autoregressive and fourier-based descriptors in $2 \mathrm{~d}$ shape classification," Pattern Analysis and Machine Intelligence, IEEE Transactions on, vol. 17, no. 2, pp. 201-207, Feb 1995.

[23] R. C. Veltkamp and M. Hagedoorn, "State of the art in shape matching," in Principles of Visual Information Retrieval, M. S. Lew, Ed. London, UK, UK: Springer-Verlag, 2001, pp. 87-119.

[24] C. Zitnick and P. Dollar, "Edge boxes: Locating object proposals from edges," in Computer Vision ECCV 2014, ser. Lecture Notes in Computer Science, D. Fleet, T. Pajdla, B. Schiele, and T. Tuytelaars, Eds. Springer International Publishing, 2014, vol. 8693, pp. 391-405.

[25] D. G. Lowe, "Distinctive image features from scale-invariant keypoints," Int. J. Comput. Vision, vol. 60, no. 2, pp. 91-110, Nov. 2004.

[26] J. Matas, O. Chum, M. Urban, and T. Pajdla, "Robust wide-baseline stereo from maximally stable extremal regions," Image and Vision Computing, vol. 22, no. 10, pp. 761 - 767, 2004, british Machine Vision Computing 2002.

[27] K. Mikolajczyk and C. Schmid, "Scale and affine invariant interest point detectors," International Journal of Computer Vision, vol. 60, no. 1, pp. 63-86, 2004.

[28] D. Nister and H. Stewenius, "Scalable recognition with a vocabulary tree," in Proceedings of the 2006 IEEE Computer Society Conference on Computer Vision and Pattern Recognition - Volume 2, ser. CVPR '06. Washington, DC, USA: IEEE Computer Society, 2006, pp. 2161-2168.

[29] J. Sivic and A. Zisserman, "Video google: a text retrieval approach to object matching in videos," in Computer Vision, 2003. Proceedings. Ninth IEEE International Conference on, Oct 2003, pp. 1470-1477 vol.2.

[30] H. Bay, A. Ess, T. Tuytelaars, and L. Van Gool, "Speeded-up robust features (surf)," Comput. Vis. Image Underst., vol. 110, no. 3, pp. 346359, Jun. 2008

[31] E. Rublee, V. Rabaud, K. Konolige, and G. Bradski, "Orb: An efficient alternative to sift or surf," in Computer Vision (ICCV), 2011 IEEE International Conference on, Nov 2011, pp. 2564-2571.

[32] K. Grauman and T. Darrell, "Efficient image matching with distributions of local invariant features," in Computer Vision and Pattern Recognition, 2005. CVPR 2005. IEEE Computer Society Conference on, vol. 2, June 2005, pp. 627-634 vol. 2.

[33] J. Philbin, O. Chum, M. Isard, J. Sivic, and A. Zisserman, "Object retrieval with large vocabularies and fast spatial matching," in Computer
Vision and Pattern Recognition, 2007. CVPR '07. IEEE Conference on, June 2007, pp. 1-8.

[34] S. Voloshynovskiy, M. Diephuis, D. Kostadinov, F. Farhadzadeh, and T. Holotyak, "On accuracy, robustness, and security of bag-of-word search systems," in IS\&T/SPIE Electronic Imaging. International Society for Optics and Photonics, 2014, pp. 902 807-902 807.

[35] J. Zhang, M. Marszalek, S. Lazebnik, and C. Schmid, "Local features and kernels for classification of texture and object categories: A comprehensive study," in Computer Vision and Pattern Recognition Workshop, 2006. CVPRW '06. Conference on, June 2006, pp. 13-13.

[36] D. Dubois, H. Prade, and F. Sedes, "Fuzzy logic techniques in multimedia database querying: a preliminary investigation of the potentials," Knowledge and Data Engineering, IEEE Transactions on, vol. 13, no. 3, pp. 383-392, May 2001.

[37] M. Everingham, L. Van Gool, C. K. I. Williams, J. Winn, and A. Zisserman, "The pascal visual object classes (voc) challenge," International Journal of Computer Vision, vol. 88, no. 2, pp. 303-338, Jun. 2010.

[38] R. T. Fielding, "Architectural styles and the design of network-based software architectures," Ph.D. dissertation, University of California, Irvine, 2000

[39] G. Bradski, "The opencv library," Doctor Dobbs Journal, vol. 25, no. 11, pp. 120-126, 2000. 